Background Sexually Transmitted Infections (STIs), including Neisseria gonorrhoeae (NG) and Chlamydia trachomatis (CT), continue to be a global health problem, with the majority of disease burden in Low-and-Middle-Income Countries. This could in part be addressed through increased access to point-of-care-tests (POCTs) to detect infection and appropriately manage cases and contacts. Criteria for the development of STI POCTs have been established, and several CT and NG POCTs have been brought to market. Yet even those diagnostics with good evidence of clinical effectiveness often fail to be implemented and adopted into routine care.

Methods We first reviewed whether the Cepheid CT/NG GeneXpert POCT fulfils published international guidance for STI POCT development: the (RE)ASSURED and Target Product Profile (TPP) criteria. Then, through a systematic review of Medline and Embase of published literature that reported on the test's implementation, demonstrated its values in different settings and to a variety of stakeholders. This information was then applied to form the basis of a value proposition for an 'ideal' CT/NG POCT.

Results The Cepheid CT/NG GeneXpert did not fulfill all (RE)ASSURED or TPP criteria, however, studies of test implementation showed multiple stakeholder values for use of the test across various healthcare settings and locations. The majority of values identified were setting-specific. Sexual health services and outreach services had the least overlap in values, whereas General Practice and other non-sexual health specialist services served as a 'bridge' between the two.

Conclusion We recommend that those wishing to improve CT/ NG diagnosis be supported to identify the values most relevant to their settings and context, and prioritise implementation of those tests most closely aligned with those values.

\section{P235 MAINTAINING ACCESS TO HIV PREP IN A PANDEMIC: PREP-USER AND HEALTH CARE PROFESSIONAL PERSPECTIVES ON A TELEPHONE-BASED PREP SERVICE}

'L Henderson*, 2,3 J Gibbs, 'S Ramasami, 'J Quinn, ${ }^{1,4} \mathrm{C}$ Estcourt. ${ }^{1} \mathrm{NHS}$ Greater Glasgow and Clyde, Glasgow, UK; ${ }^{2}$ Central and North West London NHS Trust, London, UK; ${ }^{3}$ University College London, London, UK; ${ }^{4}$ Glasgow Caledonian University, Glasgow, UK

\subsection{6/sextrans-2021-sti.317}

Background To maintain access to PrEP during the COVID-19 pandemic our PrEP service (1000 PrEP-users) shifted to a largely telephone-based model (tele-PrEP).

Objectives To conduct a service evaluation of tele-PrEP, exploring the views and experiences of PrEP-users and sexual health care professionals (HCPs), to understand benefits and drawbacks to inform future service delivery.

Methods Parallel, web-based, anonymous surveys of PrEP-users and HCPs were developed using validated questions wherever possible. The PrEP-user survey was offered to people who had a tele-PrEP appointment between 13.11.2020-17.12.2020 and consented to participate. All HCPs conducting tele-PrEP appointments were invited to participate. Basic demographic data was captured. Data were analysed in Excel using descriptive statistics. Free text responses were thematically categorised using the Framework for a Systems Approach to Healthcare Delivery.

Results Sixty-two PrEP-users and 8 HCPs completed the surveys (response rate $55 \%$ and $89 \%$ respectively). Demographic characteristics of PrEP-user respondents were broadly representative of our whole PrEP-cohort. Tele-PrEP was rated 'excellent' or 'good' by 61/62 PrEP-users, and 59/62 would recommend it to friends. PrEP-users identified convenience as a key benefit along with access to PrEP with reduced potential for COVID-19 exposure. Drawbacks were largely technological, including poor connection or issues with online booking. All HCPs felt that tele-PrEP allowed them to assess patients safely and confidently. HCPs also rated its convenience highly and felt it enabled better use of limited face-to-face clinic capacity. However, HCPs thought that tele-PrEP might create barriers for vulnerable patients, particularly those with low digital, health and/or English-language literacy. One HCP and 10/62 PrEP-users expressed a personal preference for face-toface appointments.

Conclusion Tele-PrEP is feasible and acceptable. While most respondents rated the service highly, others identified a need/ preference for face-to-face appointments. Therefore, our service will continue tele-PrEP whilst ensuring availability of faceto-face care for those who require or request it.

\section{P236 EXAMINING THE IMPACT OF THE UK'S COVID-19 PUBLIC HEALTH RESPONSE ON SEXUAL BEHAVIOUR AND HEALTH SERVICE USE AMONG MSM}

${ }^{1} \mathrm{~A}$ Howarth, ${ }^{1,2} \mathrm{~J}$ Saunders, ${ }^{1,3} \mathrm{D}$ Reid*, ${ }^{1} \mathrm{I}$ Kelly, ${ }^{1} \mathrm{~S}$ Wayal, ${ }^{3} \mathrm{P}$ Weatherburn, ${ }^{2} \mathrm{G}$ Hughes, ${ }^{1} \mathrm{C}$ Mercer. ' UCL, London, UK; ${ }^{2}$ Public Health England, London, UK; ${ }^{3}$ London School of Hygiene and Tropical Medicine, London, UK

\subsection{6/sextrans-2021-sti.318}

Background The introduction of social distancing in response to the COVID-19 pandemic led to reduced STI/HIV service provision in the UK. We investigated sexual risk behaviours among MSM and unmet need for sexual healthcare during the pandemic.

Methods A cross-sectional online survey $(\mathrm{N}=2,018)$ fielded via social media and dating apps (23/06-14/07/2020). We examined sexual behaviour and service use since lockdown (23/03/ 2020) and in the three previous months, and 'unmet need for STI testing' since lockdown (any new male partners and/or multiple condomless anal sex (CAS) partners without testing for STIs).

We compared behaviours over the past three months between socio-demographically equivalent sub-samples recruited via Grindr into the present survey $(\mathrm{N}=956)$ and a 2017 survey $(\mathrm{N}=1,918)$.

Results In 2020, 36.7\% of participants reported new male partners and $17.3 \%$ reported multiple CAS partners since lockdown. Comparing time since lockdown vs previous three months, HIV testers were less likely to test at sexual health clinics $(22.3 \%$ vs $70.2 \%)$ and more likely to use free online self-sampling services (64.3\% vs $17.1 \%)$, and PrEP users were less likely to report PrEP use (21.7\% vs $65.7 \%)$.

Since lockdown, $25.3 \%$ of participants had unmet need for STI testing. Unmet need was more likely among Asian vs White participants $(\mathrm{aOR}=1.76,[1.14-2.72], \mathrm{p}=.01)$; living in Scotland $(\mathrm{aOR}=2.02,[1.40-2.91], \mathrm{p}<.001)$ or Northern Ireland $(\mathrm{aOR}=1.93,[1.02-3.63], \mathrm{p}=.04)$ vs England; and living with HIV $(\mathrm{aOR}=1.83,[1.32-2.53], \mathrm{p}<.001)$.

Compared to 2017, the 2020 sub-sample were less likely to report new male partners $(46.8 \%$ vs $71.1 \%, \mathrm{p}<.001)$, multiple CAS partners $(20.3 \%$ vs $30.8 \%, \mathrm{p}<.001)$ and unmet need $(32.8 \%$ vs $42.5 \%, \mathrm{p}<.001)$ in the past three months. 